\title{
Etching and High-Resolution Backscatter Electron Imaging for Semi-automated Segmentation and Stereology of the Gamma Prime Phase in Ni-based Superalloys
}

\author{
E.J. Payton, ${ }^{*}$ P.J. Phillips, ${ }^{* *}$ and M.J. Mills** \\ * Institut für Werkstoffe, Ruhr-Universität Bochum, 44801 Bochum, Germany \\ ** Department of Materials Science \& Engineering, Ohio State University, Columbus, OH 43210
}

Quantitative characterization of materials microstructure is required for the development and calibration of models of microstructural evolution. Segmentation of microstructural features from images is the rate-limiting characterization task in many studies of microstructural evolution [1,2]. Additionally, recent work has indicated that the size distributions of microstructural features can play a significant role in microstructural evolution [2,3]. The present work illustrates a combination of sample preparation, imaging technique, segmentation algorithm, and stereological analysis that can be applied to Ni-based superalloys for rapid characterization of the size distribution of the gamma prime $\left(\gamma^{\prime}\right)$ phase for the study of microstructural evolution.

Specimens of René 88 DT [4] and René 104 [5] were analyzed under various microscope conditions and etched states to determine optimal imaging and sample preparation conditions for producing easily segmentable images. Imaging of $\gamma^{\prime}$-etched specimens in the immersion lens mode of an FEI Sirion XL-30 field emission scanning electron microscope using a standard pole-piece mounted backscatter detector produces strong contrast between the matrix and the $\gamma^{\prime}$ regions with sufficient spatial resolution to image tertiary $\gamma^{\prime}$ particles. A segmentation procedure found to produce high quality results with minimal user intervention for this combination of sample preparation and imaging conditions is presented in Fig. 1. The backscatter signal in the scanning electron microscope yields images that are more readily segmented with image processing algorithms than other signals, but the interaction volume of backscatter electrons complicates stereology of such images. To estimate the observation bias produced by the backscatter signal, Monte Carlo methods were used to simulate backscatter electron images, as shown in Fig. 2. The simulation results illustrate the effect of accelerating voltage on image resolution and that particles with centers beneath the sample surface appear larger than their correct planar intersection. The segmentation procedure from Fig. 1 was applied to the simulated images shown in Fig. 2 to quantify the bias of the backscatter imaging technique. The over-representation of subsurface voids was observed to be partially compensated by under-representation of voids with centers above the surface of polish. Results were incorporated into the stereological procedure used to calculate $3 \mathrm{D}$ particle size distributions from $2 \mathrm{D}$ observations.

The described combination of sample preparation, imaging technique, segmentation algorithm, and stereological correction was applied to study the evolution of $\gamma^{\prime}$ volume fraction and size during dissolution and coarsening in René 104. The results show the mean particle size to remain constant while the volume fraction drops dramatically, in excellent agreement with the prediction in [3] for an initially lognormal distribution [6].

\section{References}

[1] E.J. Payton et al. Mater. Sci. Eng. A 527 (2010) 2684.

[2] E.J. Payton. Ph.D. Thesis, The Ohio State University (2009).

[3] G. Wang et al. Acta Mater. 57 (2009) 316. 
[4] US Patent 4,957,567 (1990).

[5] European Patent EP 1,195,446 A1 (2002).

[6] Support for this work was provided by GE Aviation.

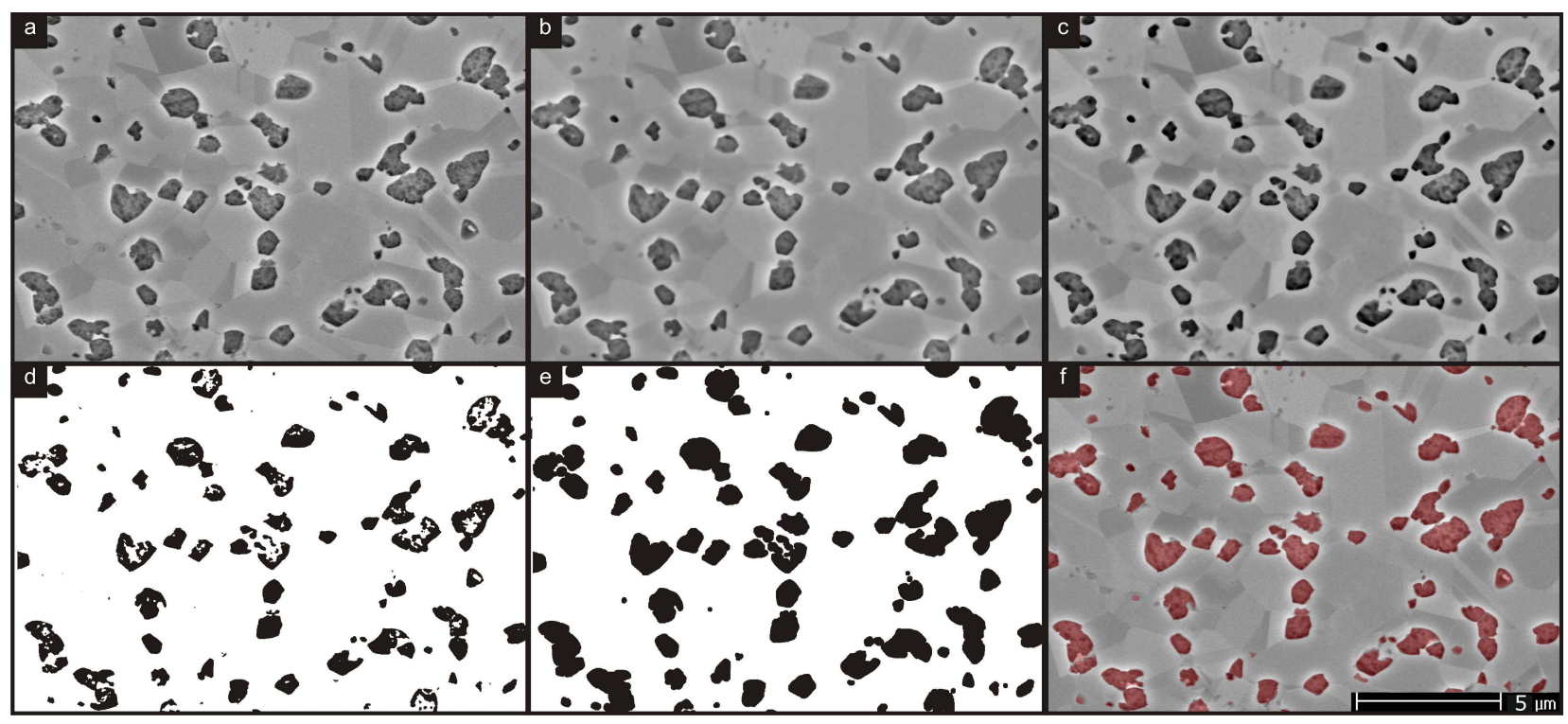

FIG. 1. Semi-automated segmentation procedure, illustrated with primary $\gamma^{\prime}$ in the same area of a specimen of René 104 imaged with a standard backscatter detector in immersion lens mode on an FEI Sirion XL-30 scanning electron microscope. (a) initial image; (b) after median filtering; (c) unsharp masking applied to (b); (d) binary threshold of (c); (e) Euclidian distance map morphology operations applied to (d); (f) overlay of final segmentation on original image.

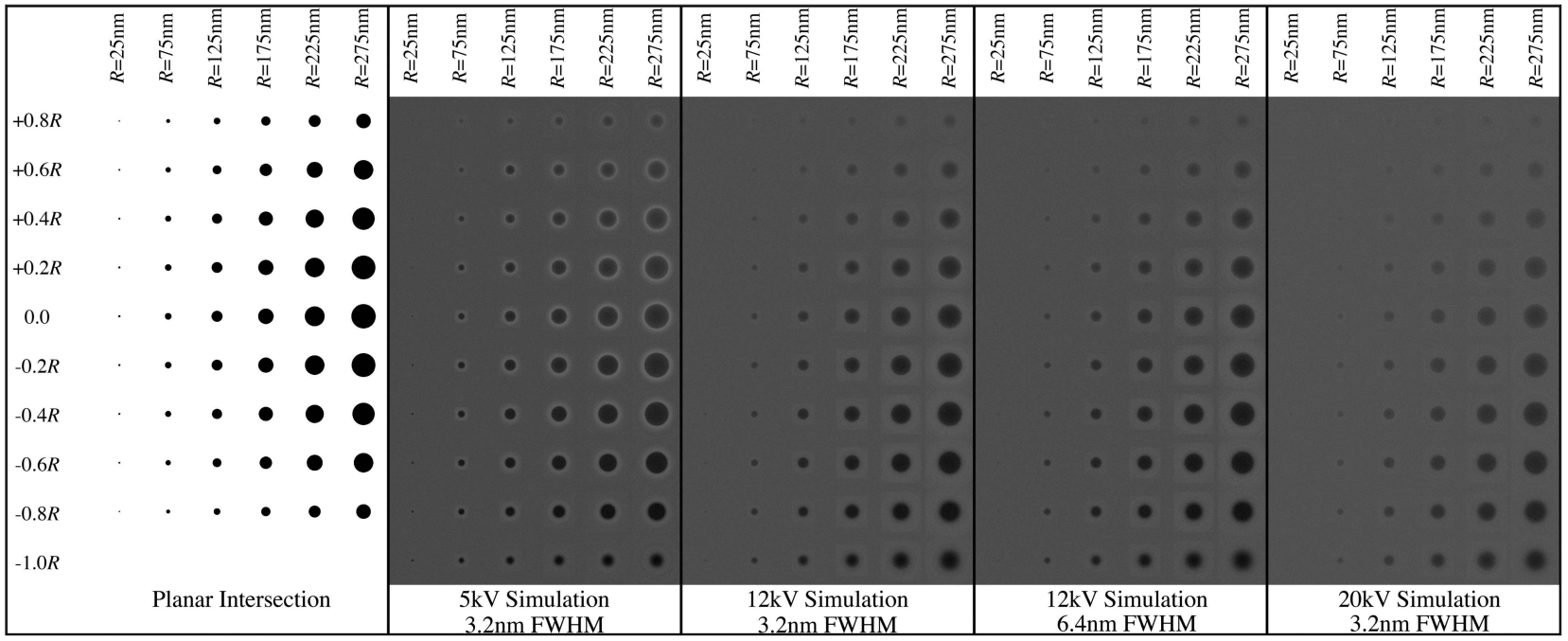

FIG. 2. Simulated backscatter images for various void sizes and depths, under different accelerating voltages and beam full widths at half-maximum, as compared with the true planar intersection. For each set of microscope conditions, voids are arranged in columns of void size and rows of void depth beneath the plane of polish. Voids with centroids beneath the surface appear larger than their correct planar intersection; increasing accelerating voltage reduces the ability to resolve small voids. 